\title{
A Giant Pleomorphic Adenoma of the Submandibular Salivary Gland: A Case Report
}

\author{
Colin J. Perumal, B.D.S. ${ }^{1}$ Mark Meyer, BChd ${ }^{2}$ Ashraf Mohamed, BChd, Mdent ${ }^{1}$ \\ ${ }^{1}$ Department of Maxillofacial and Oral Surgery, School of Oral Health \\ Sciences, University of Limpopo, Pretoria, Gauteng, South Africa \\ 2 Department of Maxillofacial and Oral Surgery, University of Western \\ Cape, Cape Town, Western Cape, South Africa \\ Address for correspondence and reprint requests Colin J. Perumal, \\ B.D.S., Department of Oral and Maxillofacial Surgery, School of Oral \\ Health Sciences, University of Limpopo, 1 Ga Rankuwa Street, \\ Ga-Rankuwa, Pretoria, Gauteng, South Africa 0204 \\ (e-mail: coljer@tiscali.co.za).
}

Craniomaxillofac Trauma Reconstruction 2012;5:185-188
Abstract
Keywords
- giant pleomorphic adenoma
- mixed tumor
- carcinoma ex pleomorphic
- adenoma
- facial deformity
- submandibular tumor

The most common benign salivary gland tumor is the pleomorphic adenoma (PA). They can attain grotesque proportions and weigh several kilograms. They can cause facial disfigurement and, if untreated, could lead to airway compromise. We report a case of a large PA arising from the left submandibular salivary gland in a 20 -year-old black female. The lesion measured $\sim 16 \times 15 \times 12 \mathrm{~cm}$.
Pleomorphic adenoma (PA) is the most common salivary gland tumor in both the major and minor salivary glands. They are also referred to as "benign mixed tumors." They represent 60 to $80 \%$ of all benign tumors in major salivary glands and 40 to $70 \%$ of minor salivary glands. ${ }^{1}$

These tumors are slow-growing and painless. However, failure to seek early treatment can result in grotesque facial deformity. $^{2}$ They are also reported to occur in the main bronchus, columella, larynx, pharynx, trachea, lacrimal gland, sinonasal tract, and maxillary sinus, albeit less commonly. ${ }^{3}$ Neck lymphadenopathy and nerve compromise are generally absent. ${ }^{2}$ The purpose of this article is to emphasize that although the PA is a well-reported entity, they can attain very large sizes and, if left untreated, could also lead to the airways being compromised. We report a case, which to the best of the author's knowledge is the largest PA of the submandibular gland.

\section{Case Report}

A 20-year-old black woman of cross-border nationality, presented to our maxillofacial and oral surgery department seeking medical attention for a large swelling on the left side of her jaw (-Figs. 1A and 1B). The swelling was painless with a history of having increased in size over an 8-year period. She noticed the swelling for the first time at the age of 12. The patient was otherwise healthy with no other significant medical history or findings.

On examination, the weight of the swelling tilted the patient's head to the left. The swelling was multinodular, nontender, hard, and mobile. Movement of the tumor mass could be elicited and palpated bimanually both intraorally (in the region of the left submandibular salivary gland) and extraorally over the mass simultaneously.

The advancing "apex" of the tumor had ulcerations and scars suggestive of previous biopsies having been done in her country of origin (-Fig. $\mathbf{2 C}$ ). The patient confirmed having had an unreported previous biopsy. Submandibular lymph nodes could not be palpated due to the tumor mass. No other supraclavicular nodes were palpable. The lesion was diagnosed clinically as a PA.

Micrograph of the incisional biopsy that was done showed features of a myxochondroid and cellular PA. The cartilage and myxoid areas grew in nodules with cellular areas in received

January 22, 2011

accepted

March 30, 2011

published online

July 27, 2012
Copyright $\odot 2012$ by Thieme Medical Publishers, Inc., 333 Seventh Avenue, New York, NY 10001, USA. Tel: +1(212) 584-4662.
DOI http://dx.doi.org/ 10.1055/s-0032-1322530. ISSN $1943-3875$. 


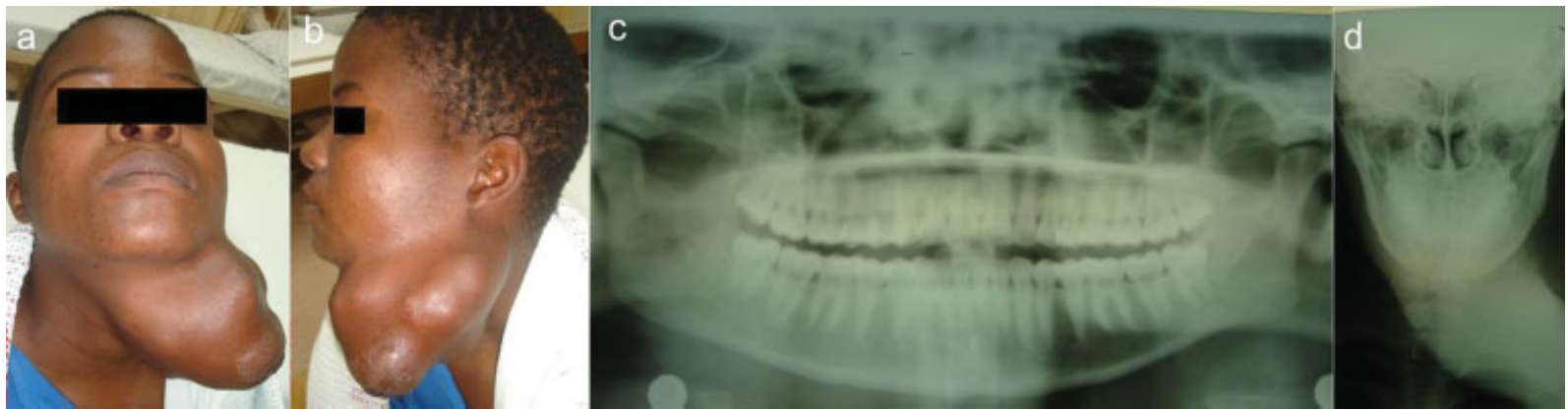

Figure 1 (A, B) Clinical presentation, front and profile views of tumor mass. (C, D) Orthopantomograph and posteroanterior views showing intact bony structures.

between. No mitotic activity was present and the tumor capsule could not be identified ( $\mathbf{- F i g . ~ 2 B}$ ). The diagnosis of PA was confirmed.

Imaging studies included an orthopantomograph, PA mandible, and computed tomography (CT; - Figs. 1A, 1D, and 2A).

CT showed a large homogenous mass involving the left submandibular space and left anterior cervical space with superior abutment of the left parotid gland. Diffuse calcifications were found within the lesion. The epicenter of the tumor was within the submandibular gland. There was medial extension to the left parapharyngeal space. Bony elements of the maxilla and mandible were within normal limits.

An en bloc resection of the tumor was done with preservation of the overlying nonulcerated skin ( - Figs. $\mathbf{2 D}$ and $\mathbf{3 A}$ to $\mathbf{3 D}$ ).
The patient recovered well and was discharged on day 4 postoperatively. Patient review was uneventful and the incision wound healed well ( - Fig. 4D).

\section{Discussion}

There is consistency in published reports showing the PA to be the commonest of the salivary gland tumors. Certainly in one of the largest studies involving 6982 primary salivary gland tumors in a Chinese population by Tian et al, PA was the most common tumor ( $69 \%$ ) of which only $20 \%$ were located in minor salivary glands. ${ }^{4}$ They have been reported to be as large as $28 \times 20 \times 16 \mathrm{~cm}$ in the parotid gland. ${ }^{5}$ Gupta et al reported weights of PAs ranging from $1 \mathrm{~kg}$ to $27 \mathrm{~kg}{ }^{6}$ The occurrence of very large PAs in the submandibular gland are
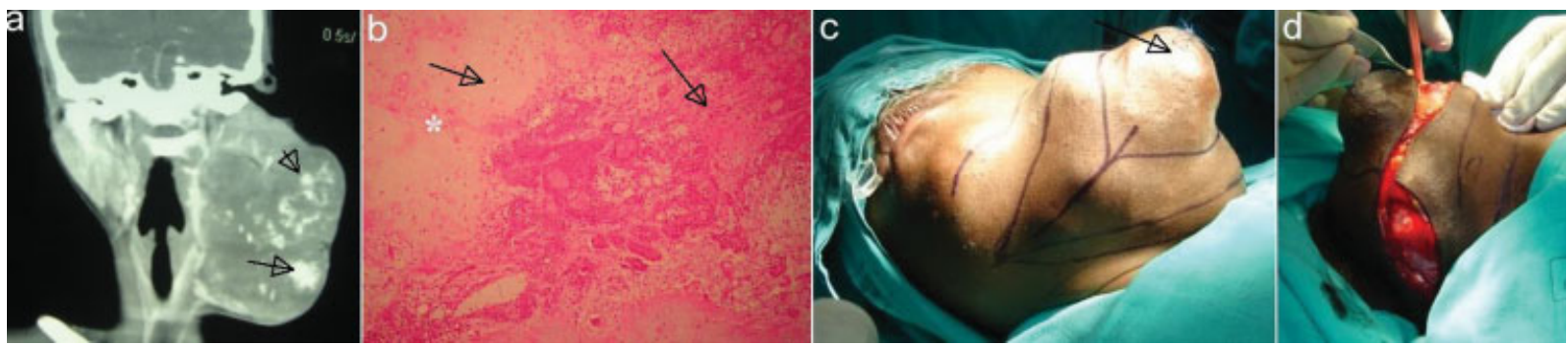

Figure 2 (A) Computed tomography scan showing the superior, inferior, and mesial extent of the tumor mass with scattered calcifications within the lesion (arrows). (B) Micrograph showing the myxochondroid and cellular nature of the tumor. (C) Arrow indicates area of sacrificed skin with ulceration (site of previous biopsy). (D) Planned marked incision line.

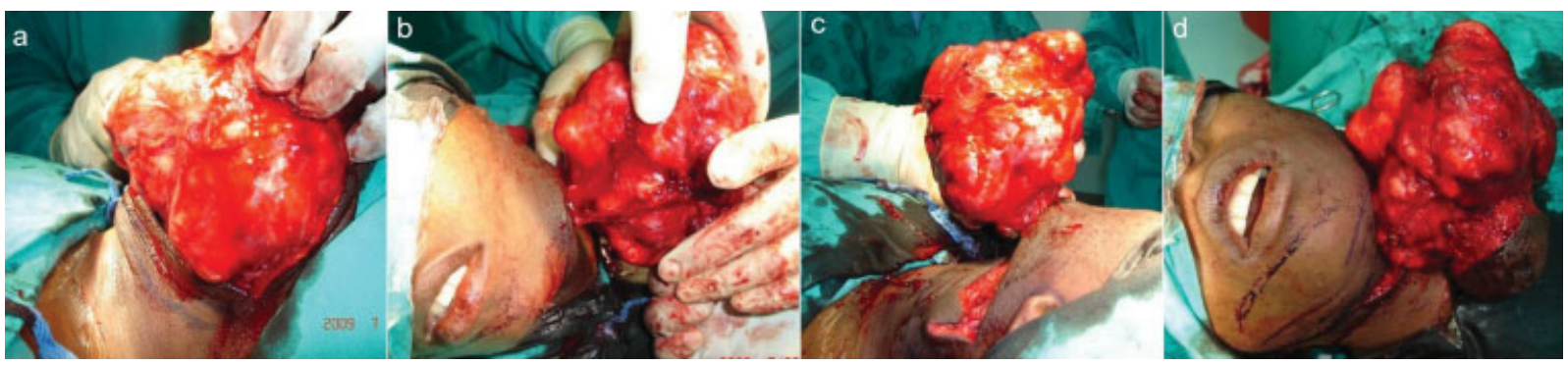

Figure 3 (A to D) Sequential surgical excision of the tumor mass. 

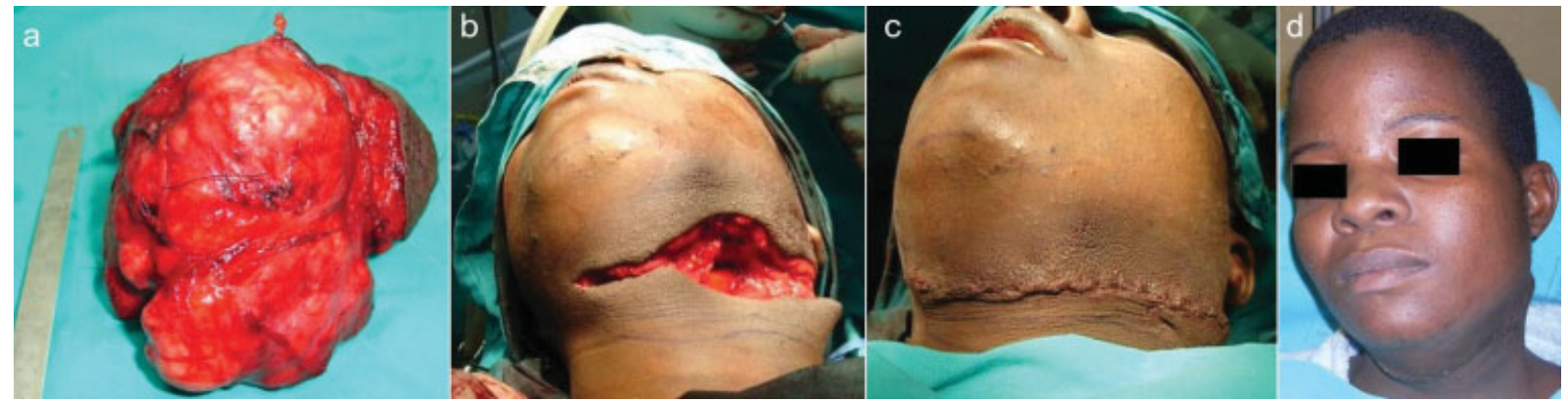

Figure 4 (A) Specimen after removal. (B) Return to almost complete aesthetic normalcy with no skin contouring being done. This demonstrates the tremendous elasticity of the skin, which had shrunk. (C, D) Wound closure and postoperative view of the patient.

rare and reports of $8 \times 6 \times 4 \mathrm{~cm}$ have been documented. ${ }^{6}$ Our case measured $16 \times 15 \times 12 \mathrm{~cm}$.

They are epithelially derived and typically present as a cytologically benign circumscribed mass with variable encapsulation. Histomorphologically, it is characterized by a variegated architecture comprising epithelial elements admixed with a mucoid, myxoid, fibrohyaline, or chondroid stoma. Historically, the main clinical problems with PA have been the risk of recurrence and progression to a clinically or histologically based malignancy. ${ }^{7}$

The rate of tumor recurrence seems to depend on surgical techniques used as this could contribute to intrasurgical rupture and tumor spillage with resultant seeding of tumor cells. Histopathologic features may also impact on the rate of recurrence. In a study of 225 patients with PA, Henriksson et

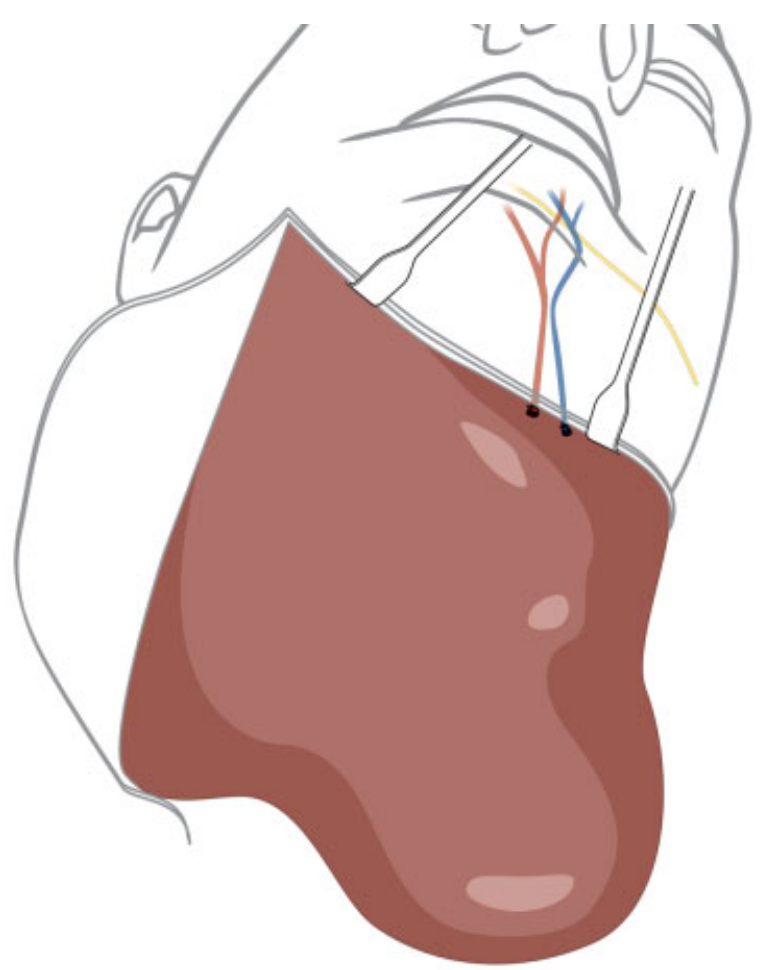

Figure 5 A sketch diagram showing the anterior view of the tumor mass and the superoanterior extent of the facial vein and artery as well as the possible region of the terminal branches of the marginal mandibular branch of the facial nerve. al found $56 \%$ of the PAs that had recurred showed fingerlike tumor extensions or pseudopodia outside the pseudocapsule microscopically as compared with the $25 \%$ of recurrences in patients who had intraoperative rupture of the tumor. ${ }^{8}$

Over recent years, there has emerged a rare but nonetheless well-documented subgroup of PA entities that require additional awareness and precise recognition in terms of their propensity for future aggressiveness. These include features of vascular invasion, focal dysplasia, and noninvasive (intracapsular) carcinoma ex pleomorphic adenoma. ${ }^{7}$

Generally, malignant transformation can be suspected with a sudden increase in size accompanied by local signs of malignancy, pain, ulceration, spontaneous bleeding, and superficial and deep tissue invasion. Furthermore, multiple recurrences and the size of the tumor may play a role in the malignant transformation of the PA. The clinical characteristics of malignant transformation have been reported in the literature as (1) a long history of PA, (2) advanced age, (3) location in a major salivary gland, and (4) history of rapid growth associated with pain or ulceration. ${ }^{7}$ Age in our case was to the patient's advantage. Representative microscopic sampling of large PAs is important to rule out malignant change, which could increase in frequency with large tumors as malignant sites could be missed.

Reporting a case of this nature has significance as delayed intervention allows for rapid tumor growth (believed by some to be due to mutated ras genes ${ }^{9}$ ), poor aesthetics, psychological trauma, and a possible threat to life especially with the presence of tumor pressure over the parapharyngeal space and oropharynx, which could be compounded by infection thus threatening the airway. The expanding mass could further distort the anatomy thus displacing vital structures and making the dissection more challenging. The possible location of nerves and blood vessels are depicted in (- Fig. 5). The presentation of cases such as the one being reported highlights the plight of many patients especially in the developing nations. Whatever the reasons; such disease progression should never be condoned, certainly not in the twenty-first century.

\section{Acknowledgments}

I thank Professor E.J. Raubenheimer, Head of Department Oral Pathology at the School Of Oral Health Sciences, 
Medunsa Campus, University of Limpopo, South Africa, for providing the histopathology slides and report.

Competing interests: None declared.

Funding: None.

Ethical approval: Not required.

\section{References}

1 Kakimoto N, Gamoh S, Tamaki J, Kishino M, Murakami S, Furukawa SCT. CT and MR images of pleomorphic adenoma in major and minor salivary glands. Eur J Radiol 2009;69:464-472

2 Molina EJ, Mayer K, Khurana J, Grewal H. Pleomorphic adenoma of the submandibular gland. J Pediatr Surg 2008;43:1224-1226

3 Ceylan A, Celenk F, Poyraz A, Uslu S. Pleomorphic adenoma of the nasal columella. Pathol Res Pract 2008;204:273-276

4 Tian Z, Li L, Wang L, Hu Y, Li J. Salivary gland neoplasms in oral and maxillofacial regions: a 23-year retrospective study of 6982 cases in an eastern Chinese population. Int J Oral Maxillofac Surg 2010;39:235-242

5 Takahama A Jr, da Cruz Perez DE, Magrin J, de Almeida OP, Kowalski LP. Giant pleomorphic adenoma of the parotid gland. Med Oral Patol Oral Cir Bucal 2008;13:E58-E60

6 Gupta M, Chaudhary N. Giant presentation of pleomorphic adenoma in major salivary gland. BMJ Case Reports 2011

7 Ethunandan M, Witton R, Hoffman G, Spedding A, Brennan PA. Atypical features in pleomorphic adenoma-a clinicopathologic study and implications for management. Int J Oral Maxillofac Surg 2006;35:608-612

8 Henriksson G, Westrin KM, Carlsöö B, Silfverswärd C. Recurrent primary pleomorphic adenomas of salivary gland origin: intrasurgical rupture, histopathologic features, and pseudopodia. Cancer 1998;82(4):617-620

9 Milašin J, Pujić N, Dedović N, et al. H-ras gene mutations in salivary gland pleomorphic adenomas. Int J Oral Maxillofac Surg 1993; 22:359-361 